\title{
CROSSOVER FROM BCS TO BOSE SUPERCONDUCTIVITY: A FUNCTIONAL INTEGRAL APPROACH*
}

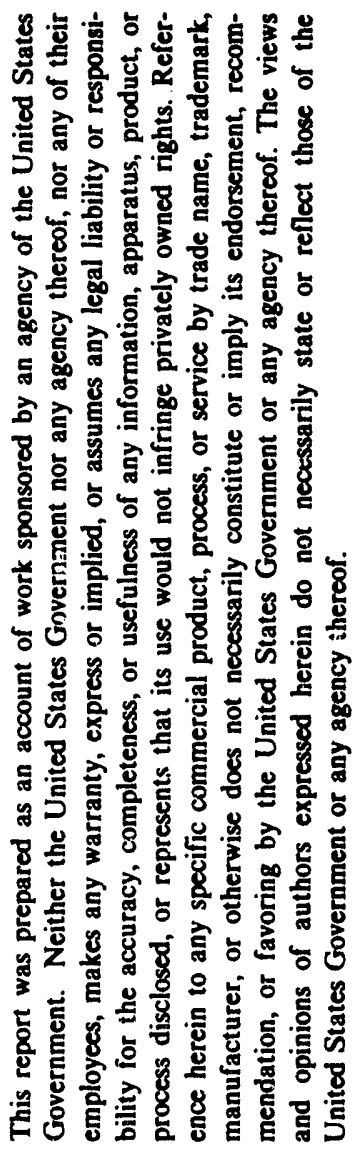

$/$ rlp
M. Randeria, C. A. R. Sa de Melo

Materials Science Division

Argonne National Laboratory

Argonne, IL 60439

J. R. Engelbrecht

Physics Department

University of Illinois-Urbana

1110 W. Green Street

Urbana, IL 61801

The submitted manuscript has been authored
by a contractor of the U. S. Government
under contract No. W-31-109-ENG-38.
Accordingly, the U.S. Government retains a
nonexclusive, royalty-free license to publish
or reproduce the published form of this
contribution, or allow others to do so, for
U.S. Government purposes.

APRIL 1993

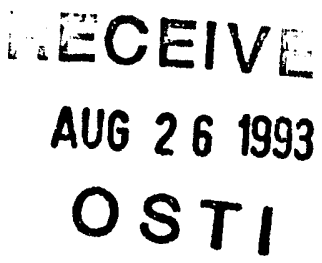

Submitted to International Conference LT XX on Low Temperature Physics, Eugene, OR, August 4-11, 1993.

* Work Supported by the U.S. Department of Energy, BES-Materials Sciences, under Contract \#W-31-109-ENG-38. 


\title{
CROSSOVER FROM BCS TO BOSE SUPERCONDUCTIVITY: A FUNCTIONAL INTEGRAL APPROACH
}

\author{
Mohit Randeria ${ }^{a}$, C. A. R. Sá de Melo ${ }^{a}$ and Jan R. Engelbrecht ${ }^{b}$ \\ a Materials Science Division, Argonne National Laboratory, Argonne IL 60439 \\ ${ }^{b}$ Physics Department, University of Illinois at Urbana-Champaign, $1110 \mathrm{~W}$. Green St, Urbana IL 61801
}

We use a functional integral formulation to study the crossover from cooperative Cooper pairing to the formation and condensation of tightly bound pairs in a $3 \mathrm{D}$ continuum model of fermions with attractive interactions. The inadequacy of a saddle point approximation with increasing coupling is pointed out, and the importance of temporal (quantum) fluctuations for normal state properties at intermediate and strong coupling is emphasized. In addition to recovering the Nozières-Schmitt-Rink interpolation scheme for $T_{c}$, and the Leggett variational results for $T=0$, we also present results for evolution of the time-dependent Ginzburg-Landau equation and collective mode spectrum as a function of the coupling.

PACS numbers: 74 .

The problem of the crossover $[1,2]$ from BCS theory with cooperative Cooper pairing to the formation and condensation of composite bosons has attracted considerable attention [3-5] after the discovery of the high $T_{c}$ superconductors which have pair sizes comparable to the average carrier separation. In this paper we summarize some of the results of an extensive analysis [6] of this problem in a 3D continuum model of fermions (mass $m$, Fermi energy $\epsilon_{F}$ ) with attraction characterized by an $s$-wave scattering length $a_{s}$.

We use a standard imaginary-time functional integral formulation at temperature $\beta^{-1}$ and decouple the fermion interaction with a pair field $\Delta$. The effective action, written in terms of the Nambu propagator $\mathbf{G}$, is then given by

$$
S_{\mathrm{eff}}=\frac{1}{g} \int_{0}^{\beta} d \tau \int d \mathbf{x}|\Delta(x)|^{2}-\operatorname{Tr} \ln \mathrm{G}^{-1}[\Delta(x)] .
$$

The bare attraction $g$ is replaced [3] by the scattering length $a_{3}$.

First we consider a saddle-point estimate of the transition temperature $T_{0}$, defined by the solution of $\delta S_{\text {eff }} / \delta \Delta[\Delta=0]=0$, and $N=-\partial \Omega / \partial \mu$. The saddle point approximation for the thermodynamic potential, $\Omega_{0}=S_{\text {eff }}[\Delta=0] / \beta$, then allows one to solve for $T_{0}$ and the chemical potential $\mu$ as a function of the coupling. In the weak coupling $g \rightarrow 0$ limit, $\left(k_{F} a_{s}\right)^{-1} \rightarrow-\infty$ and we find the $\mathrm{BCS}$ results $\mu \simeq \epsilon_{F}$ and $T_{0} \simeq$ $8 e^{-2} \gamma \pi^{-1} \epsilon_{F} \exp \left(-\pi / 2 k_{F}\left|a_{s}\right|\right)$.

However, the saddle-point approximation becomes progressively worse with increasing coupling. In the strong coupling $g \rightarrow \infty$ limit $\left(k_{F} a_{s}\right)^{-1} \rightarrow+\infty$ and one finds tightly bound pairs with binding energy $E_{b}=1 / m a_{s}^{2}$. The nondegenerate Fermi system has $\mu \simeq-E_{b} / 2$ and $T_{0} \simeq\left(E_{b} / 2\right) / \ln \left(E_{b} / \epsilon_{F}\right)^{3 / 2}$. This result is an artifact of the approximation and the strong coupling $T_{0}$ is related to the pair dissociation scale rather than the $T_{c}\left(\ll T_{0}\right)$ at which coherence is established.

We next include the effects of gaussian fluctuations using $S_{\text {gauss }}=S_{\text {eff }}[\Delta=0]$ $+\sum_{\mathbf{q}, i q_{m}} \Gamma^{-1}\left(\mathbf{q}, i q_{m}\right)\left|\Delta\left(\mathbf{q}, i q_{m}\right)\right|^{2}$, with $\Gamma^{-1}=$ $1 / g-\chi_{\text {pair }}^{0}$, to obtain the $\Omega$ used in the number equation $N=-\partial \Omega / \partial \mu$. While the weak coupling results are unaffected, the strong coupling results are completely changed by the appearance of a bound state pole in $\Gamma$. Within a pole approximation the $g \rightarrow \infty$ partition function reduces to that of a free Bose gas (mass $2 m$ and density $n / 2$ ) with the Bose condensation $T_{c}=[n / 2 \zeta(3 / 2)]^{2 / 3} \pi / m$.

We see that the bosonic degrees of freedom, which become important with increasing $g$, can only be adequately described by retaining the full frequency dependent gaussian fluctuations in the normal state. Numerical calculations show that 
these quantum fluctuations affect $T_{c}$ significantly even in the intermediate coupling regime. The gaussian theory for $T_{c}$ is identical to the NozièresSchmitt-Rink interpolation [2]. Going beyond this approximation we find a $\Delta^{4}$ term, which for $g \rightarrow \infty$ represents a two-body repulsion between the composite bosons as shown below.

We next turn to the evolution of the timedependent Ginzburg-Landau (TDGL) equation near $T_{c}$ from the BCS to the Bose regime. We first expand $S_{\text {eff }}$ to order $\Delta^{4}$ and then in powers of $|q|$ and $\omega$ (after analytically continuing $\left.i q_{m} \rightarrow \omega+i 0^{+}\right)$. On either side of the point $\mu\left(T_{c}\right)=0$ (see below) one has a well defined TDGL equation, for $\varepsilon=\left(T-T_{c}\right) / T_{c} \ll 1$, provided $\Delta \ll|\omega| \ll \min \left(T_{c}, \mu\left(T_{c}\right)\right)$.

In the BCS regime we obtain the usual TDGL with characteristic length scale $\xi(T)=\xi_{0} \varepsilon^{-1 / 2}$ and time scale $\tau(T)=\tau_{0} \varepsilon^{-1}$ with $\xi_{0} \simeq v_{F} / T_{c}$ ( $\sim \xi_{\text {pair }}$, the pair size), and $\tau_{0} \simeq 1 / T_{c}$. The width of the Ginzburg region is $\left(T_{c} / \epsilon_{F}\right)^{4}$. The dynamics of the order parameter is overdamped reflecting the continuum of fermionic excitations into which a pair can decay. There is in addition a small $\mathcal{O}\left(T_{c} / \epsilon_{F}\right)$ propagating part since the model is not particle-hole symmetric.

With increasing coupling the propagating piece grows while the damped part diminishes. A singular point is obtained at an intermediate coupling where $\mu\left(T_{c}\right)=0$. The vanishing energy scale does not allow a low frequency expansion and even a linearized TDGL description fails.

Beyond this singular point an essentially propagating mode is obtained. In the extreme strong coupling Bose limit the TDGL reduces to the Gross-Pitaevskii equation for a dilute Bose gas. The repulsion between bosons is characterized by a (boson) scattering length $a_{b}=2 a_{s}>0$ with $n_{b} a_{b}^{3} \ll 1$, where $n_{b}=n / 2 \sim k_{F}^{3}$ (in terms of the $k_{F}$ of the constituent fermions). The prefactors of the divergent length and time scales at the transitions are given by $\xi_{0} \simeq k_{F}^{-1} / \sqrt{k_{F} a_{s}} \gg \xi_{\text {pair }} \simeq a_{s}$ and $\tau_{0} \simeq T_{c}^{-1} /\left(k_{F} a_{s}\right)$ respectively. These are both much longer than the microscopic scales because of the diluteness condition $k_{F} a_{s} \ll 1$.

We obtain the following results for the broken symmetry state. The saddle point gap and num- ber equations at $T=0$ are identical to Leggett's variational equations [1]. The non-trivial saddle point of $S_{\text {eff }}$ includes the non-perturbative effects of bound state formation and condensation and we find that, at least for $T \ll T_{c}$, the gaussian fluctuations make only a small correction even for strong coupling (in marked contrast to the normal state discussed above).

The collective excitation spectrum at $T=0$ is obtained from the gaussian fluctuations. For the charge neutral system considered here ve find a sound mode $\omega=c q$ for long wavelengths where explicit formulae have been obtained for $c$, and the eigenvector corresponding to this mode, as function of the coupling. In the two extreme limits we obtain the analytical results. In the BCS limit $c=v_{F} / \sqrt{3}$ corresponding to the AndersonBogoliubov result, which evolves smoothly into the Bogoliubov mode for the dilute Bose gas with $c=\left(\pi n a_{2} / m^{2}\right)^{1 / 2}$. Note that the repulsion between bosons discussed above is crucial for the collective mode spectrum. Also, unlike the regime close to $T_{c}$ with its singular point when $\mu\left(T_{c}\right)=0$, the low frequency expansion for $T \ll T_{c}$ is well defined for all couplings, since a gap in the spectrum precludes a vanishing energy scale, and that the sound mode evolves smoothly from one limit to another.

We gratefully acknowledge support from grants NSF STC 88-09854 (CSdM), DOE W-31-109ENG-38 (MR), and NSF DMR 88-22688 (JRE).

[1] A. J. Leggett in "Modern Trends in the Theory of Condensed Matter", Edited by A. Pekalski and R. Przystawa, (Springer, 1980).

[2] P. Nozières and S. Schmitt-Rink, J. Low Temp. Phys. 59, 195 (1985).

[3] M. Randeria, J. Duan, and L. Shieh, Phys. Rev. Lett. 62, 981 (1989); Phys. Rev. B41, 327 (1990).

[4] M. Randeria, N. Trivedi, A. Moreo, and R. T. Scalettar, Phys. Rev. Lett. 69, 2001 (1992).

[5] M. Drechsler and W. Zwerger, Ann. Physik 1, 15 (1992).

[6] C. A. R. Sá de Melo, M. Randeria, and J. R. Engelbrecht (in preparation). 

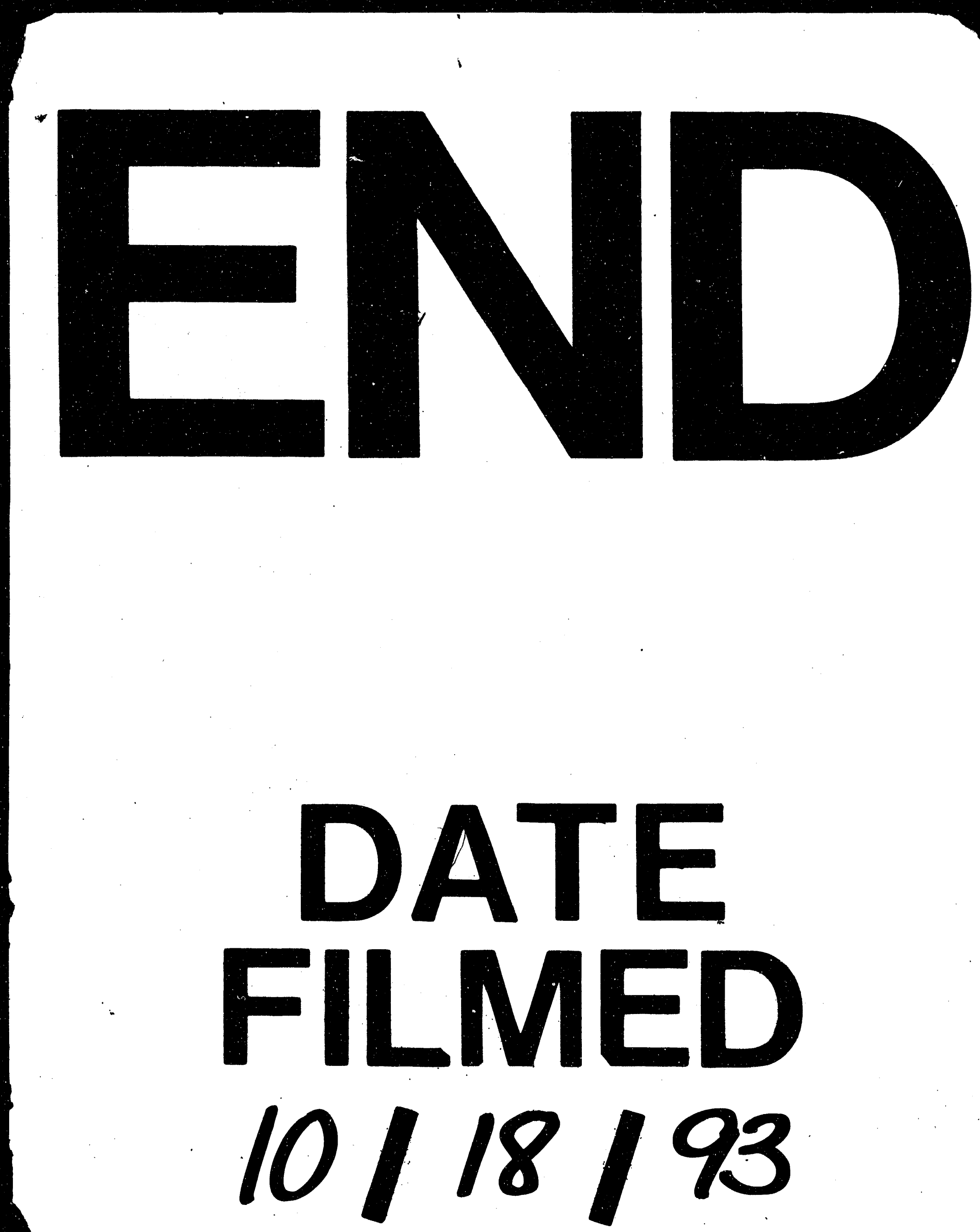
